\title{
Surface Electromyography Assessments of the Vastus medialis and Rectus femoris Muscles and Creatine Kinase after Eccentric Contraction Following Glutamine Supplementation
}

\author{
Farhad Rahmani-Nia ${ }^{\text {1ACDEFG }}$, PhD; Esmail Farzaneh ${ }^{\text {*2ABCDEG }}$, MSc; Arsalan Damirchi ${ }^{\text {1CDEG }}$, PhD;
} Ali Shamsi Majlan ${ }^{1 C D E G}$, PhD; Vahid Tadibi ${ }^{3 \mathrm{CDEG}}$, PhD

\author{
Authors' Affiliation: \\ 1. Faculty of Physical Education and Sport \\ Sciences, University of Guilan, Rasht, \\ Iran \\ 2. Department of Physical Education and \\ Sport Sciences, Kermanshah Branch, \\ Islamic Azad University, Kermanshah, \\ Iran \\ 3. Faculty of Physical Education, Razi \\ University, Kermanshah, Iran

$$
\begin{aligned}
& \text { Authors' Contribution } \\
& \text { A Concept / Design } \\
& \text { C Data Analysis / Interpretation } \\
& \text { E Critical Revision of the Manuscript } \\
& \text { F Funds Collection } \\
& \text { G Approval of the Article }
\end{aligned}
$$ \\ * Corresponding Author; \\ Address: Department of Physical Education \\ and Sport Sciences, Kermanshah Branch, \\ Islamic Azad University, Kermanshah, Iran \\ E-mail: esmail_farzaneh@yahoo.com
}

Received: Apr 04, 2013

Accepted: Nov 03, 2013

Available Online: Nov 29, 2013

\begin{abstract}
Purpose: L-glutamine is the most abundant amino acid found in human muscle and plays an important role in protein synthesis and can reduce the levels of inflammation biomarkers and creatine kinase (CK) after training sessions. Delayed onset muscle soreness (DOMS) develops after intense exercise and is associated with an inflammatory response. The purpose of this study was to investigate the effect of glutamine supplementation on surface electromyography activity of the vastus medialis muscle (VMM) and rectus femoris muscle (RFM) and levels of creatine kinase after an eccentric contraction.
\end{abstract}

Methods: Seventeen healthy men (age: 22.35 $\pm 2.27 y$; body mass: 69.91 \pm 9.78kg; height: $177.08 \pm 4.32 \mathrm{~cm}$ ) were randomly assigned to experimental $(n=9)$ and control groups $(n=8)$ in a double-blind manner. In both groups, subjects were given L-glutamine supplementation $\left(0.1{\mathrm{~g} . \mathrm{kg}^{-1}}^{-1}\right.$ or placebo three times a week for 4 weeks. Median frequency (MDF) and mean power frequency (MPF) for VMM and RFM muscles and also CK measurements were performed before, $24 \mathrm{~h}$ and $48 \mathrm{~h}$ after a resistance training session. The resistance training included 6 sets of eccentric leg extensions to exhaustion with $75 \%$ of $1 R M$.

Results: There was no significant difference between groups for MDF or MPF in VMM and RFM. The difference of CK level between the groups was also not significant.

Conclusion: The results of this study indicate that glutamine supplementation has no positive effect on muscle injury markers after a resistance training session.

Key Words: Mean Power Frequency; Median Frequency; Creatine Kinase; Eccentric Exercise

Asian Journal of Sports Medicine, Volume 5 (Number 1), March 2014, Pages: 54-62

\section{INTRODUCTION}

$\mathrm{T}$ he quadriceps femoris plays an important role in explosive and powerful actions of the leg during sport and daily activities ${ }^{[1]}$. Eccentric contraction is commonly used during training programs to improve muscular power for the quadriceps muscles ${ }^{[1]}$. However, eccentric exercise (EE) of the quadriceps is associated with fiber injury and Delayed onset muscle soreness (DOMS) and therefore may reduce the ability of the quadriceps to stabilize the knee joint. This may expose structures of the knee to abnormal load during exercise. It has been shown that DOMS can decrease muscle activation as is reflected by reduction of electromyographic (EMG) activity during post eccentric sustained isometric contraction ${ }^{[2,3]}$.

The reduced muscle activation may be caused by abnormal afferent input from damaged and inflamed tissue of the injured muscle ${ }^{[4]}$ that affects the excitability of alpha and gamma motor neurons. In 
addition, Morgan and Allen (1999) thought the changes in organization of the sarcomere structure and changes in excitation-contraction coupling appear to be the main contributors to the early reduction in tension ${ }^{[5]}$. Surface electromyography (sEMG) has become a common method used in athlete technical analysis ${ }^{[6]}$, muscle fiber type identification ${ }^{[7]}$ and muscle fatigue research ${ }^{[8]}$. Additionally, some scholars suggest that sEMG can indicate muscle damage by selective recruitment of different motor units ${ }^{[9,10,11]}{ }_{\text {._SEMG is a }}$ technique for evaluating and recording physiologic properties of muscles at rest and during exercise ${ }^{[12]}$. One popular measure of EMG frequency content is median frequency (MDF: the point at which the spectral power is divided into equal low and highfrequency halves) and mean power frequency (MPF: characteristics of frequency of EMG). The EMG power spectrum is shifted towards the lower frequencies as exemplified by the fall in MPF during static contractions as well as during dynamic exercise. The EMG spectrum from eccentric, concentric muscle contractions and increased serum levels of muscle proteins such as creatine kinase (CK) in humans were studied as a factor of mechanical damage of muscle fiber and functional change of metabolic tissue [13,14].

Deschenes et al (2000) reported that the impairment in neuromuscular efficiency outlasted other symptoms of damage such as strength loss, muscle soreness, and increased circulating levels of myofiber proteins ${ }^{[15]}$.

Prophylactic and therapeutic nutritional interventions involving protein, protein hydrolysate, mixed amino acids, selective amino acids, and branched-chain amino acids have been demonstrated to be effective in reducing some or all of the symptoms of muscle damage following isolated eccentric muscle actions ${ }^{[16,17,18]}$, resistance exercise ${ }^{[19]}$, downhill running ${ }^{[20]}$, and endurance exercise ${ }^{[21]}$.

The underlying mechanism by which protein or amino acid supplementation can attenuate symptoms of muscle damage is not fully understood. Greater amino acid availability ${ }^{[17,18]}$, extra energy intake from supplementation ${ }^{[17]}$, increased protein synthesis and/or decreased protein breakdown ${ }^{[19,20]}$ have been suggested as potential underlying mechanisms.

Glutamine is the most abundant amino acid within the human body and is synthesized de novo from glutamate and ammonia in a wide variety of tissues containing glutamine synthetase. It is considered as a nonessential amino acid, however can be consider as a "conditionally essential" amino acid; because, its plasma concentration decreases up to $50 \%$ after a stressful activity ${ }^{[22,23]}$.

During exercise, increase or decrease in plasma glutamine level has been demonstrated and these variations are reflected by the type, duration, and intensity of exercise ${ }^{[24]}$. Evidence regarding the influence of eccentric exercise induced muscle damage on plasma glutamine concentration is equivocal ${ }^{[25,26]}$. Recently, it is showed that an acute reduction in muscle glutamine concentration two hours following a resistance exercise, including 40 leg-press repetitions at $80 \%$ of one repetition maximum ${ }^{[27]}$. Glutamine supplementation has been proposed as a possible ergogenic aid for maximizing strength gains following resistance training. More specifically, glutamine supplementation is thought to prevent protein degradation and therefore enhance recovery following intense resistance-training sessions ${ }^{[28]}$. Recently, Cruzat et al (2010) concluded that supplementation with free L-glutamine and the dipeptide alanylglutamine represents an effective source of glutamine, which may attenuate inflammation biomarkers after periods of training and plasma levels of CK and the inflammatory response induced by prolonged exercise in rats ${ }^{[29]}$. In addition, street et al (2011) administered $0.3 \mathrm{~g} . \mathrm{kg}^{-1} \mathrm{~L}$-glutamine at $0,24,48$, and 72 hours postexercise and found that glutamine supplementation was effective in attenuating strength loss and muscle soreness following eccentric exercise-induced muscle damage ${ }^{[30]}$.

Therefore, potential exists for glutamine homeostasis to be disturbed following eccentric exercise, manifested as a reduction in muscle and/or plasma glutamine concentration ${ }^{[26,27]}$. In such a context, oral glutamine supplementation may restore glutamine homeostasis and maintain a positive net protein balance that can reduce the inflammatory response to eccentric exercise resulting in attenuation of exercise-induced muscle damage and affect indirect markers such as strength loss, soreness, MDF, MPF and CK activity. 
Clinical intravenous doses of glutamine are typically $20-25 \mathrm{~g}$ per day ${ }^{[31]}$, and exercise studies have employed oral ingestion doses of up to $45 \mathrm{~g}$ per day for 6 weeks without adverse effects ${ }^{[32,33]}$.

Currently, there is little research regarding measuring indices of muscle damage when a glutamine supplementation is administered prior to eccentric exercise. Therefore, the purpose of this study was to investigate the potential effect of glutamine supplementation on surface electromyographic activity and levels of $\mathrm{CK}$ after an eccentric contraction.

\section{METHODS AND SUBJECTS}

\section{Subjects:}

Twenty healthy college-aged untrained men between 19-25 years of age volunteered with informed consent to participate in this study. Subjects were free from musculoskeletal injuries and had not taken any dietary supplement within the past 6 months before the beginning of the study. Subjects were instructed to avoid therapeutic treatment of any symptoms of muscle damage and to maintain their normal diet throughout the experiment. Seventeen participants, who fully completed the treatment and protocols, were considered as the subjects of this study (Fig. 1). They were randomly assigned into glutamine and placebo group in a double-blind manner.

\section{Experimental design and exercise protocol:}

One week prior to the first experimental trial, subjects attended a familiarization session where by demonstrations of the testing procedures were provided with subjects undertaking no eccentric exercise. Testing was conducted at the exercise physiology laboratory of faculty of physical education at Guilan University. The experimental design consisted of baseline testing followed by repeated testing of the same parameters at 24 and 48 hours after the muscledamaging exercise. After the baseline measurement of plasma CK and EMG activity (MDF, MPF), subjects performed a resistance training session including eccentric leg extension movement (6 set to exhaustion with $75 \%$ of $1 \mathrm{RM}$ ) using leg extension machine. The rest intervals between the sets were 3 minutes ${ }^{[34]}$. Subjects received verbal encouragement throughout the protocol to maintain maximal effort.

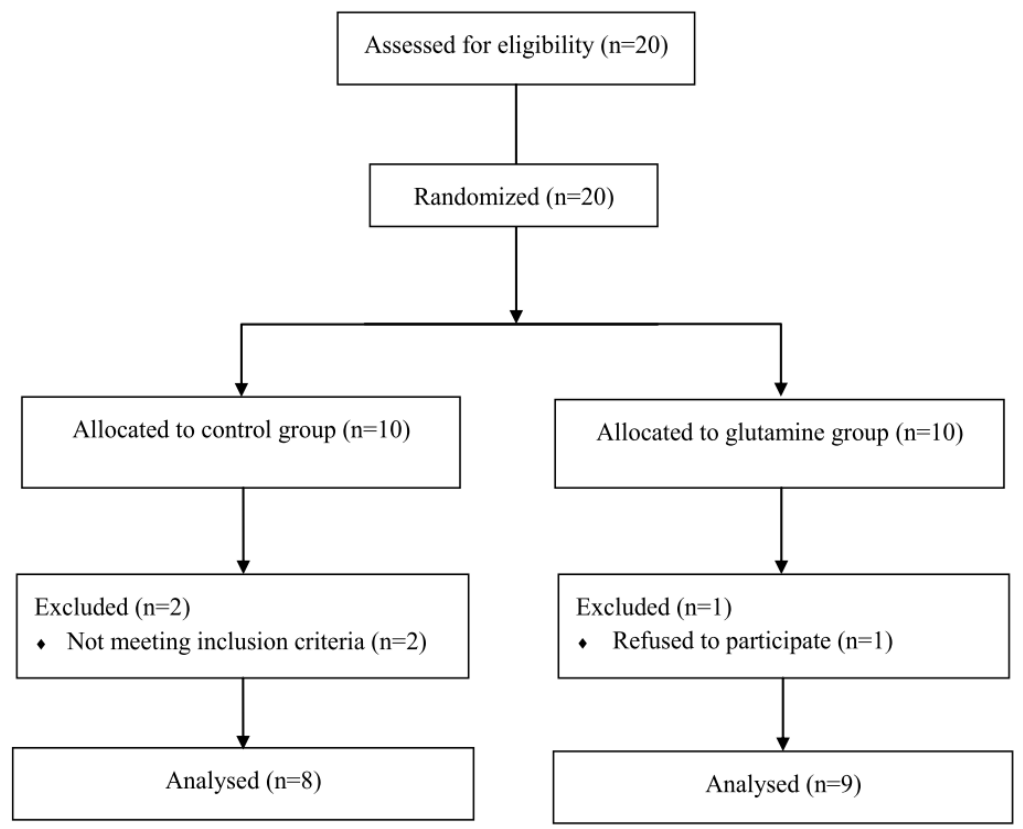

Fig. 1: Study flow diagram 


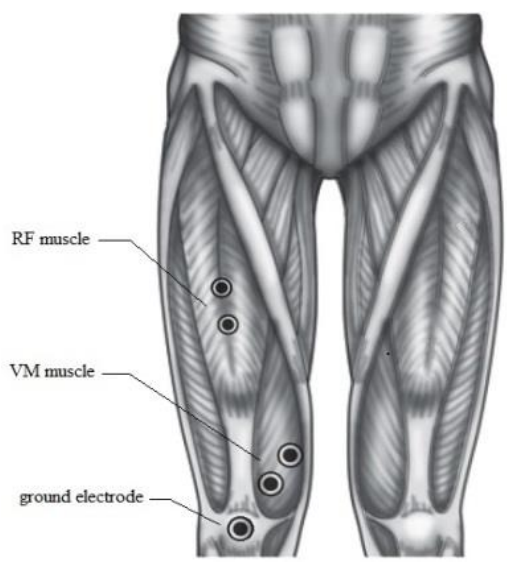

Fig. 2: Electrode placement for the RF (Location: This muscle is located on the center of the anterior surface of the thigh, approximately half the distance between the knee and the iliac spine. The two active electrodes are placed $2 \mathrm{~cm}$ apart, parallel to the muscle fibers), VM (Location: The placement for VM oblique is done using 2-cm spacing, with the electrodes placed at an oblique angle (55 degrees), $2 \mathrm{~cm}$ medially from the superior rim of the patella. Palpate for the muscle during extension of the knee. It is somewhat easier to palpate toward the end of the range of motion. The electrodes are placed on the distal third of the VM) and ground electrode site. (VM: vastus medialis)

\section{Diet assessment:}

Each subject was screened for dietary habits before inclusion into the study. Subjects completed 7-d diet records 1-d in each week and 3-d during the training period, randomly. After the 4-d diet record participants, received dietary counseling by a registered dietitian to ensure that the participants maintained their habitual dietary habits and ingested the supplement three times a week and assured adherence to all supplement and food instructions. Food diaries were analyzed for energy and macro/micronutrient content with NUTRITIONIST IV (Version 3.5.2).

\section{Supplementation:}

In both groups, subjects took L-glutamine supplementation $\left(0.1 \mathrm{~g} \cdot \mathrm{kg}^{-1}\right.$; Karen pharma \& food supplement. co. Iran) or placebo (Maltodextrin) mixed with about $500 \mathrm{ml}$ of water after lunch three times a week for 4 weeks.

\section{Measurements:}

EMG recordings: The subjects performed three maximal voluntary isometric contractions (MVC) separated by 2-min rest. sEMG of the vastus medialis muscle (VMM) and rectus femoris muscle (RFM) was measured during $\mathrm{MVC}$, the participants were in a seated position with restraining straps across the chest and hip with the right knee $90^{\circ}$ flexed. The sEMG was recorded bipolarly by electrodes (silver-silver chloride electrodes) with an inter electrode distance of $20 \mathrm{~mm}$ over the VMM and RFM at four sites on the right legs and the ground electrode was placed over the patella, each landmark was identified using anatomical landmarks (Fig. 2). Before electrode placement, the skin was lightly abraded. The electrodes were placed longitudinally on the muscle belly on each marked site. EMG activity was assessed by Mega Win ME3000P8 device (MEGA Electronics, Ltd, Finland) and analyzed by Mega Win 2.01. EMG parameters have been used to evaluate muscle damage and recovery after eccentric exercise. Amongst them, MDF, MPF have been used frequently in previous studies ${ }^{[14,24,35,36,37]}$.

$C K$ : At each sampling time, about 5-ml of venous blood was withdrawn from the antecubital vein, centrifuged for 10-min to extract plasma samples, and analysed for CK activity within 24h. Serum CK activity was measured in duplicate using an enzymatic assay kit (Pars azmun; Tehran, Iran).

\section{Statistical Analysis:}

The statistical analysis was initially done using the changes in the One-Sample Kolmogorov-Smirnov test. All variables showed normal distribution. Two-way 
Table 1: Physical characteristics of the subjects

\begin{tabular}{lcc} 
Variable & $\begin{array}{c}\text { Placebo group }(\mathbf{n}=\mathbf{8}) \\
\text { Mean (SD) }\end{array}$ & $\begin{array}{c}\text { Glutamine group }(\mathbf{n}=\mathbf{9}) \\
\text { Mean (SD) }\end{array}$ \\
\hline age $(\mathbf{y r})$ & $22.4(2.3)$ & $22.3(2.3)$ \\
height $(\mathbf{c m})$ & $176.5(5.6)$ & $177.7(3.0)$ \\
weight $(\mathbf{k g})$ & $72.2(11.0)$ & $67.6(8.5)$ \\
Body Fat $(\%)$ & $14.5(3.5)$ & $12.7(2.9)$ \\
Body Mass Index $\left(\mathbf{k g . m ^ { 2 }}\right)$ & $22.8(2.5)$ & $21.4(2.4)$
\end{tabular}

SD: Standard Deviation

analysis of variance with repeated measurement was used to compare differences in MPF, MDF and CK between Glutamine and Control group at different time points. Significant main effects were followed by Bonferroni. Independent t-test was used to compare time points (before, 24h, 48h) in Glutamine and Control group. Significance was accepted when $P<0.05$. SPSS 16.0 Statistical package was used for analyzing data.

\section{RESULTS}

\section{Characteristics of subjects:}

The general characteristics of the remaining 17 subjects are shown in Table 1. There were no differences among groups for age, body weight, height, body fat percentage and body mass index (Table 1).

\section{Changes in SEMG after eccentric exercise:}

MDF and MPF for VMM and RFM were not different between groups (Table 2). As shown in Fig. $3 \mathrm{~A}_{1}$ and $\mathrm{B}_{1}$, there were significant time-by-Control group interaction effects for MDF for VMM and RFM muscles, but there was no significant difference between $24 \mathrm{~h}$ and $48 \mathrm{~h}$. Fig. $3 \mathrm{~A}_{1}$ and $\mathrm{B}_{1}$ showed a significant decrease in $\mathrm{MDF}$ during $\mathrm{MVC}$ after eccentric exercise, which recovered afterwards. Whereas in Glutamine group there was significant time interaction effect for MDF for RFM but in VMM there was significant difference only between $24 \mathrm{~h}$ and before $(P \leq 0.05)$.

In addition, as shown in Fig. $3 \mathrm{~A}_{2}$ and $\mathrm{B}_{2}$, the MPF in VMM and RFM muscle decreased during MVC in each group, but gradually returned towards the initial values 48 hours after eccentric exercise. MPF for VMM and RFM muscle in Control group with MDF for VMM and RFM muscle in Control group showed similar patterns. Nonetheless, there were significant time-by-Glutamine group interaction effects for MPF for VMM and RFM $(P \leq 0.05)$.

\section{Changes in serum CK after eccentric exercise:}

CK values increased across time for two groups from baseline (Fig. 4). However no significant differences were found between groups at any point in time (Table 2).

Table 2: difference between groups regarding research variables between times

\begin{tabular}{lcccc} 
Variable & $\begin{array}{c}\text { Significant difference } \\
\text { at }(\boldsymbol{P} \leq \mathbf{0 . 0 5}) \text { Level }\end{array}$ & $\begin{array}{c}\text { Mean } \\
\text { Difference }\end{array}$ & $\boldsymbol{T}$. value & $\boldsymbol{P}$. value \\
\hline $\begin{array}{l}\text { Median frequency - vastus } \\
\text { medialis muscle }\end{array}$ & Pre-24 & -4.44 & -1.62 & 0.1 \\
Median frequency - rectus & Pre-48 & -4.90 & -1.24 & 0.2 \\
femoris muscle & Pre-24 & -3.47 & -1.64 & 0.1 \\
Mean power frequency - vastus & Pre-48 & -8.28 & -4.08 & 0.06 \\
medialis muscle & Pre-24 & 0.33 & 0.23 & 0.8 \\
Mean power frequency - rectus & Pre-48 & -1.86 & -2.13 & 0.08 \\
femoris muscle & Pre-24 & 1.05 & 0.60 & 0.6 \\
Creatine kinase & Pre-48 & -1.30 & -0.91 & 0.4 \\
& Pre-24 & 17.04 & 0.76 & 0.5 \\
& Pre-48 & 31.81 & -1.82 & 0.09
\end{tabular}




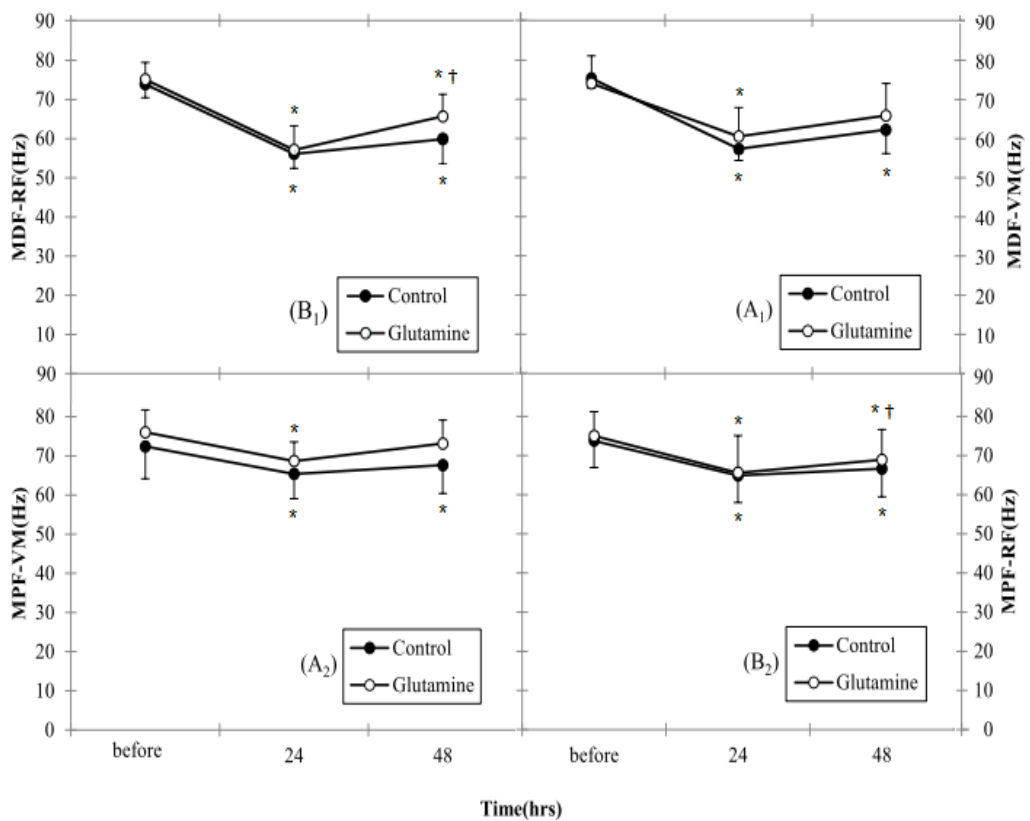

Fig. 3: $\left(A_{1}\right)$ Surface EMG index, MDF for VM in leg extension before and after EE. $\left(B_{1}\right)$ Surface EMG index, MDF for RF in leg extension before and after EE. $\left(\mathrm{A}_{2}\right)$ Surface EMG index, MPF for VM in leg extension before and after EE. $\left(\mathrm{B}_{2}\right)$ Surface EMG index, MPF for $\mathrm{RF}$ in leg extension before and after EE. *Denotes significant difference from before EE,

$\dagger$ Denotes significant difference from 24 hour after EE. $P \leq 0.05$. Data are mean \pm Standard Error. (EMG: electromyography; MDF: Median frequency; VM: vastus medialis; RF: rectus femoris; EE: eccentric exercise )

\section{DISCUSSION}

The purpose of this study was surface electromyography (MDF, MPF) assessments of the VMM and RFM and CK levels after eccentric contraction following glutamine supplementation.

It has been claimed that in athletes subjected to some types of exercise, particularly intense and prolonged exercise, chronic inflammatory process, hyperalgesia, an elevated number of injuries and DOMS are observed. These side effects of exercise are detrimental to health, hamper the cellular repair process and compromise athletic performance ${ }^{[16]}$. Exercise-induced muscle damage have been evaluated both directly ${ }^{[38]}$ and indirectly ${ }^{[39]}$. However, indirect methods of evaluating muscle damage have been preferred, and these have generally been utilized in human studies. Some examples of indirect methods have included measuring changes in plasma CK activity, inflammatory markers (in plasma and muscles) and neuromuscular function (measured by
EMG) ${ }^{[40]}$.

A possible mechanism of glutamine action is attenuating the inflammatory response to eccentric exercise. A local inflammatory response is observed in skeletal muscle post eccentric exercise ${ }^{[41]}$, with the timing and magnitude of leukocyte infiltration associated with decrements in muscle function ${ }^{[42]}$. Glutamine is known to maintain protein balance and plays many cell regulatory and immune system roles [31]. Glutamine supplementation may improve muscle function by alleviating the local inflammatory response to eccentric exercise ${ }^{[42]}$.

In our study, the activity of CK increased across time for two groups from baseline; showed no significant difference between groups at any point in time. These results disagreed with Cruzat et al (2010) which found that glutamine supplementation lowered plasma CK ${ }^{[29]}$. One possible explanation for discrepancies seen may be related to the form of ingested amino acids. We administered free form amino acids, while Cruzat et al administered alanyl- 


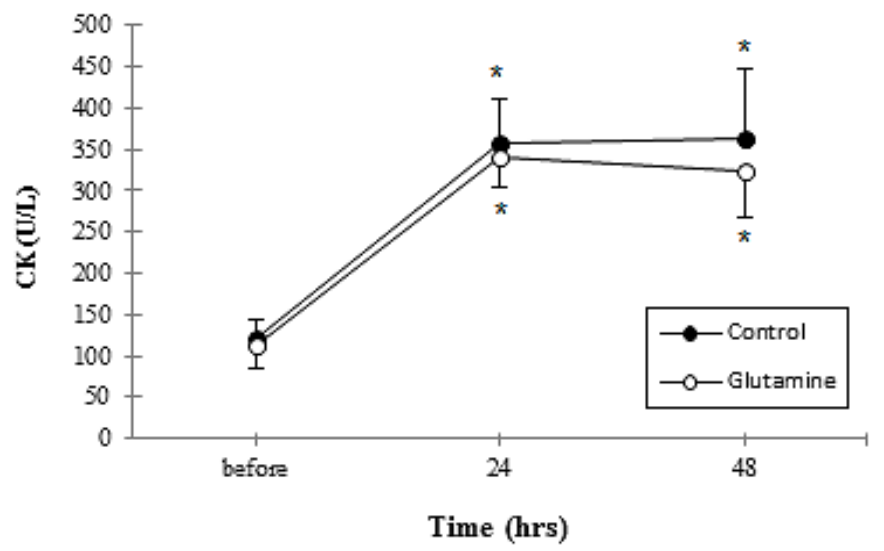

Fig. 4: Serum creatine kinase concentration before and after eccentric exercise. *Denotes significantly different from before eccentric exercise. $P \leq 0.05$. Data are mean \pm Standard Error

glutamine. It is possible that glutamine derivatives such as alanyl-glutamine may be more stable than glutamine itself, especially at low $\mathrm{pH}^{[43]}$. A second explanation may be related to the dose of glutamine contained in the protein administered in the present study. However, these findings partially agreed with Street et al ${ }^{[30]}$ who found that L-glutamine supplementation did not affect the magnitude of the $\mathrm{CK}$ response.

Also, our data shows that MDF and MPF were decreased in the each group for VMM and RFM after 24 hours. Reduction in MPF and MDF after the eccentric exercise is consistent with Chen et al ${ }^{[39]}$ and Linnamo et al ${ }^{[44]}$. This decrease could be explained by several mechanisms. For example, a possible reason is that some muscle fibers were inhibited by ultrastructure damage after eccentric contraction, and this impaired the neuromuscular function ${ }^{[14]}$. Also, elevated blood lactate concentration and increased proton $\left(\mathrm{H}^{+}\right)$ accumulation may be partly responsible for the decrease in MDF. In the absence of blood lactate an impairment of the excitation-contraction coupling has been suggested to be responsible for the changes in MDF ${ }^{[44]}$ and the selective injury of the fast twitch fibers after eccentric exercise ${ }^{[45]}$.

But EMG activity of RFM in Glutamine group greatly increases after 24 hours compared to Control group. However, The RFM has the greater number of Type II fibers followed by the VMM. This difference may be attributed to the effects of glutamine supplementation in increasing the amount of protein synthesis by reducing degradation that can increase the size of fast twitch fibers, enhancing buffering capacity. ${ }^{[46]}$ Also, the transport of glutamine to the cell interior is an active process, which is dependent upon sodium $\left(\mathrm{Na}^{+}\right)$, and promotes, concomitantly, water absorption by the cell and potassium $\left(\mathrm{K}^{+}\right)$release from the cell ${ }^{[25,}$ ${ }^{26]}$. This mechanism increases the hydration state of the cell and influences its volume, which results in increased resistance of the cell to lesions, less release of intracellular enzymes like CK and a less intense inflammatory process ${ }^{[26,27]}$.

\section{CONCLUSION}

The decrease of MPF, MDF in the VMM and RFM and increase of CK levels identified immediately after the eccentric exercise, indicates a failure in the mechanism of muscle fiber contraction, probably associated with muscle fiber damage and from these results, our study indicates that glutamine supplementation has no significant effect on muscle injury markers in between groups. Although glutamine supplementation attenuated EMG activity of RF muscle in Glutamine group, however differences in muscle cross-sectional area, skin thickness beneath the EMG electrode, and cross talk from neighboring muscles can also modify some of these same EMG activities. 


\section{ACKNOWLEDGMENTS}

The authors wish to thank the subjects for their participation in this study.

\section{Conflict of Interest: None}

\section{REFERENCES}

[1] Jones DA, Rutherford OM. Human muscle strength training: the effects of three different regimens and the nature of the resultant changes. J Physiol 1987;391:1-11.

[2] Hagberg C, Hagberg M. Surface EMG amplitude and frequency dependence on exerted force for the upper trapezius muscle: a comparison between right and left sides. Eur J Appl Physiol Occup Physiol 1989;58:641-5.

[3] Pincivero DM, Gandhi V, Timmons MK, et al. Quadriceps femoris electromyogram during concentric, isometric and eccentric phases of fatiguing dynamic knee extensions. J Biomech 2006;39:246-54.

[4] Hurley MV. The effects of joint damage on muscle function, proprioception and rehabilitation. Man Ther 1997;2:11-7.

[5] Morgan DL, Allen DG. Early events in stretch-induced muscle damage. J Appl Physiol 1999;87:2007-15.

[6] Pieter Clarys J, Scafoglieri A, Tresignie J, et al. Critical Appraisal and Hazards of Surface Electromyography Data Acquisition in Sport and Exercise. Asian J Sports Med 2010;1:69-80.

[7] Gerdle B, Karlsson S, Crenshaw AG, et al. The influences of muscle fibre proportions and areas upon EMG during maximal dynamic knee extensions. Eur J Appl Physiol 2000;81:2-10.

[8] Taylor JL, Gandevia SC. A comparison of central aspects of fatigue in submaximal and maximal voluntary contractions. $J$ Appl Physiol 2008;104:542-50.

[9] McHugh MP, Connolly DA, Eston RG, et al. Electromyographic analysis of exercise resulting in symptoms of muscle damage. $J$ Sports Sci 2000;18:163-72.

[10] Felici F, Colace L, Sbriccoli P. Surface EMG modifications after eccentric exercise. J Electromyogr Kines 1997;73:193-202.

[11] Sbriccoli P, Felici F, Rosponi A, et al. Exercise induced muscle damage and recovery assessed by means of linear and non-linear sEMG analysis and ultrasonography. J Electromyogr Kines 2001;112:73-83.

[12] Kamen G, Caldwell GE. Physiology and interpretation of the electromyogram. J Clin Neurophysiol 1996;13:366-84.

[13] Merletti R, Lo Conte L. Sathyan D. Repeatability of Electrically-evokedMyoelectric Signals in the Human Tibialis Anterior Muscle. $J$ Electromyogr Kines 1995;5:67-80.

[14] Zhou Y, Li Y, Wang R. Evaluation of exercise-induced muscle damage by surface electromyography. J Electromyogr Kines 2011;21: 356-62.

[15] Deschenes MR, Brewer RE, Bush JA, et al. Neuromuscular disturbance outlasts other symptoms of exercise-induced muscle damage. J Neurol Sci 2000;174:92-9.

[16] Buckley JD, Thomson RL, Coates AM, et al. Supplementation with a whey protein hydrolysate enhances recovery of muscle forcegenerating capacity following eccentric exercise. J Sci Med Sport 2010;13:178-81.

[17] Jackman SR, Witard OC, Jeukendrup AE, et al. Branched-chain amino acid ingestion can ameliorate soreness from eccentric exercise. Med Sci Sports Exerc 2010;42:962-70.

[18] Nosaka K, Sacco P, Mawatari K. Effects of amino acid supplementation on muscle soreness and damage. Int J Sport Nutr Exerc Metab 2006;16:620-35

[19] Kraemer WJ, Ratamess NA, Volek JS, et al. The effect of amino acid supplementation on hormonal responses to resistance training overreaching. Metabolism 2006;55:282-91.

[20] Etheridge T, Philp A, Watt PW. A single protein meal increases the recovery of muscle function following an acute eccentric exercise bout. Appl Physiol Nutr Metab 2008;33:1-6.

[21] Greer BK, Woodard JL, White JP, et al. Branched-chain amino acid supplementation and indicators of muscle damage after endurance exercise. Int J Sport Nutr Exerc Metab 2007;17:595-607.

[22] Matilla B, Ortíz J, González P, et al. Effects of parenteral nutrition supplemented with glutamine or glutamine dipeptides on liver antioxidant and detoxication systems in rats. Nutrition 2000;16:125-8.

[23] Curi R, Lagranha CJ, Doi SQ, et al. Molecular mechanisms of glutamine action. J Cell Physiol 2005;204:392-401.

[24] Eriksson LS, Broberg S, Bjorkman O, et al. Ammonia metabolism during exercise in man. Clin Physiol 1985;5:325-36.

[25] Gleeson M, Walsh NP, Blannin AK, et al. The effect of severe eccentric exercise-induced muscle damage on plasma elastase, glutamine and zinc concentrations. Eur J Appl Physiol 1998;77:543-6.

[26] Miles MP, Naukam RJ, Hackney AC, et al. Blood leukocyte and glutamine fluctuations after eccentric exercise. Int J Sports Med 1990;20:322-7. 
[27] Blomstrand E, Essen-Gustavsson B. Changes in amino acid con-centration in plasma and type I and type II fibres during resistance exercise and recovery in human subjects. Amino Acid 2009;37:629-36.

[28] Antonio J, Street C. Glutamine: A potentially useful supplement for Athletes. Can J Appl Physiol 1999;24:1-14.

[29] Cruzat VF, Rogero MM, Tirapegui J. Effects of supplementation with free glutamine and the dipeptide alanyl-glutamine on parameters of muscle damage and inflammation in rats submitted to prolonged exercise. J Cell Biochem Funct 2010;28:24-30.

[30] Street B, Byrne Ch, Eston R. Glutamine Supplementation in Recovery From Eccentric Exercise Attenuates Strength Loss and Muscle Soreness. JESF 2011;9:116-122

[31] Wernerman J. Clinical use of glutamine supplementation. J Nutr 2008;138:2040-4.

[32] Gleeson M. Dosing and efficacy of glutamine supplementation in human exercise and sport training. J Nutr 2008;138:2045-9.

[33] Candow DG, Chilibeck PD, Burke DG, et al. Effect of glutamine supplementation combined with resistance training in young adults. Eur J Appl Physiol 2001;86:142-9.

[34] Stock MS, Young JC, Golding LA, et al. The effects of adding leucine to pre and postexercise carbohydrate beverages on acute muscle recovery from resistance training. J Strength Cond Res 2010;24:2211-9.

[35] McHugh MP, Connolly DA, Eston RG, et al. Electromyographic analysis of repeated bouts of eccentric exercise. J Sports Sci 2001; 19:163-70.

[36] Lowery Lonnie, Forsythe Cassandra E. Protein and Overtraining: Potential Applications for Free-Living Athletes. J Int Soc Sports Nutr 2006;3:42-50.

[37] Alfonsi E, Pavesi R, Merlo IM, et al. Hemoglobin near-infrared spectroscopy and surface EMG study in muscle ischaemia and fatiguing isometric contraction. J Sports Med Physiol Fitness 1999;39:83-92.

[38] Stauber WT, Clarkson PM, Fritz VK, et al. Extracellular matrix disruption and pain after eccentric muscle action. J Appl Physiol 1990;69:868-74.

[39] Chen C, Trevor. Effects of a second bout of maximal eccentric exercise on muscle damage and electromyographic activity. Eur J Appl Physiol 2003;89:115-21.

[40] Clarkson P, Hubal M. Exercise-induced muscle damage in humans. Am J Phys Med Rehabil 2002;81(Suppl. 11):52-69.

[41] Peake J, Nosaka N, Suzuki K. Characterization of inflammatory responses to eccentric exercise in humans. Exerc Immunol Rev 2005;11:64-85.

[42] Paulsen G, Crameri R, Benestad HB, et al. Time course of leukocyte accumulation after eccentric exercise. Med Sci Sports Exerc 2010;42:75-85.

[43] Fürst P. New developments in glutamine delivery. J Nutr 2001;131(suppl):2562-8.

[44] Linnamo V, Bottas R, Komi PV. Force and EMG power spectrum during and after eccentric and concentric fatigue. $J$ Electromyogr Kinesiol 2000;10:293-300.

[45] Lieber RL, Fridén J. Mechanisms of muscle injury after eccentric contraction. J Sci Med Sport 1999;2:253-65.

[46] Phillips GC. Glutamine: the nonessential amino acid for performance enhancement. Curr Sports Med Rep 2007;6:265-8. 